A Platinum Open Access Journal for Organic Chemistry

Paper

Archive for

Organic Chemistry

DOAJ Seal

Arkivoc 2021, part viii, 167-178

\title{
1,3,4-Thiadiazol-2-ylphenyl-1,2,4,5-tetrazines: efficient synthesis via Pinner reaction and their luminescent properties
}

\author{
Anna Maj,, Agnieszka Kudelko, ${ }^{a^{*}}$ and Marcin Świątkowski ${ }^{b}$
}

${ }^{a}$ The Silesian University of Technology, Department of Chemical Organic Technology and Petrochemistry, Krzywoustego 4, PL-44100 Gliwice, Poland

${ }^{b}$ Lodz University of Technology, Institute of General and Ecological Chemistry, Zeromskiego 116, PL-90924 Lodz, Poland

Email: Agnieszka.Kudelko@polsl.pl

Received 01-21-2021

Accepted 04-16-2021

Published on line 06-06-2021

\section{Abstract}

Due to the interest in, and diverse applications of, 1,2,4,5-tetrazines and 1,3,4-thiadiazoles, individually and, more recently, in combination, a series of novel 1,2,4,5-tetrazine derivatives conjugated directly or via a 1,4phenylene linker with a 1,3,4-thiadiazole core, were synthesized. A six-step reaction sequence, involving the Pinner reaction and oxidation under mild conditions, was used. This approach worked well for both symmetrical and unsymmetrical arrangements. Their luminescence properties were examined and are reported. The obtained compounds may have a number of great applications potential.
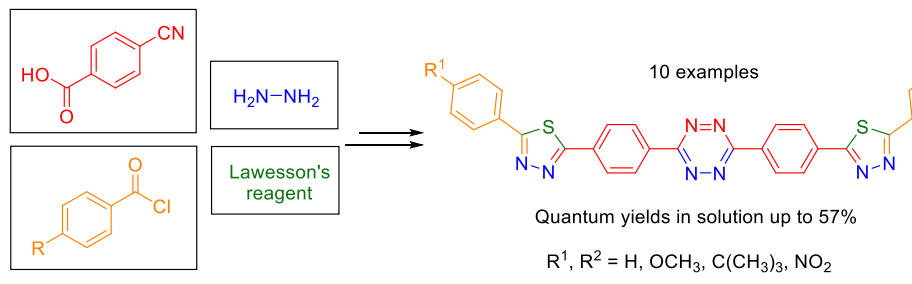

Keywords: Tetrazines, 1,2,4,5-tetrazine, 1,3,4-thiadiazole, Pinner reaction, Lawesson's reagent, aromatic carbonitriles 


\section{Introduction}

One of the most interesting heterocyclic arrangements, characterised by both the maximum content of nitrogen atoms and its ring stability, is the six-membered 1,2,4,5-tetrazine (s-tetrazine). ${ }^{1}$ Among its most commonly described applications, one has to distinguish high-energy-density materials (HEDM), which allow their utilisation in the production of explosives and propellants. ${ }^{2}$ However, the derivatives of this compound also have great potential for applications in other fields. In the literature, there are more and more studies on the possibilities of using s-tetrazine derivatives in laser dyes, organic light emitting diodes (OLEDs) or perovskite solar cells. ${ }^{3-5}$ Optoelectronics is an intensively developing and researched field, however, new organic $n$-type semiconductors, which would allow improvements in the efficiency and durability of devices, are still being researched. Accordingly, nitrogen-containing compounds are of great interest. Due to the high electronegativity of nitrogen, heterocycles rich in this atom are characterised by a large electron deficit. The use of such systems allows the modification of the charge-transport properties of a given material to obtain the appropriate levels of bandgap energies. In addition to $s$-tetrazine derivatives, examples of systems that work perfectly in this role are compounds containing 1,3,4-thiadiazole..$^{6,7}$ Its derivatives can also be used as high-performance wide-bandgap copolymer donors for efficient non-fullerene organic solar cells. ${ }^{8}$

Both s-tetrazine and 1,3,4-thiadiazole also exhibit a broad spectrum of biological activity. Consequently, their derivatives can be used in medicine ${ }^{9-19}$ and 1,3,4-thiadiazole-containing systems serve as plantprotection products. ${ }^{20,21}$ In addition, an extremely interesting potential application of $s$-tetrazine derivatives is in medical diagnostics. These compounds are excellent substrates in Diels-Alder reactions with inverse electron demand used in bioorthogonal chemistry. ${ }^{22-24}$

The combination of $s$-tetrazine and 1,3,4-thiadiazole seems to be very promising as well, especially for applications in optoelectronics. However, very few types of derivatives have been described in the literature so far. In our previous work, we synthesised a series of $s$-tetrazines directly conjugated to 1,3,4-thiadiazoles which exhibited excellent fluorescent properties. ${ }^{25}$ We were interested in how the introduction of the 1,4phenylene linker between the heterocyclic rings would affect the luminescent properties of the new compounds.

The work reported here describes a convenient and practical methodology for the synthesis of 3,6-bis(4(1,3,4-thiadiazol-2-yl)phenyl)-1,2,4,5-tetrazine derivatives from aromatic carbonitriles, hydrazine hydrate and Lawesson's reagent. We present another series of compounds containing these two rings, this time connected via a 1,4-phenylene linker. We were also interested in the results due to the possibility of comparing the properties of the obtained compounds with analogous systems containing 1,3,4-oxadiazole. ${ }^{26}$ To the best of our knowledge, the preparation and fluorescent properties of the title compounds, containing an extended $\pi$ conjugated system, have not been previously reported.

\section{Results and Discussion}

The title 1,2,4,5-tetrazine derivatives conjugated via a 1,4-phenylene linker to a 1,3,4-thiadiazole ring were obtained in a six-step reaction sequence. The first four steps were aimed at the formation of a five-membered ring without compromising the stability of the carbonitrile moiety (Scheme 1). For this purpose, commercially available 4-cyanobenzoic acid (1) was used. The specific structure of this compound allows its application as a linker between 1,2,4,5-tetrazine and 1,3,4-thiadiazole. In the first step, the carboxyl group present in the substrate was subjected to esterification using methanol and hydrochloric acid, which gave ester $\mathbf{2}$. The next 
step was the conversion of compound $\mathbf{2}$ by reaction with hydrazine hydrate in methanol which resulted in product 3. In order to form the necessary diacylhydrazine moiety, as well as to introduce an additional ring with substituents of various types, hydrazide $\mathbf{3}$ was reacted with freshly prepared aroyl chlorides $\mathbf{4 a - d}$. A series of products, 5a-d, were obtained. The derivatives thus prepared were reacted with Lawesson's reagent in dry toluene. At this stage, the influence on the reaction yield of the type of substituents introduced by the aroyl chlorides was evident. The presence of electron-donor groups (methoxy and tert-butyl) led not only to higher yields, but also to a reduction in the heating time (5b, 5c, entries 2 and 3, Table 1$)$.

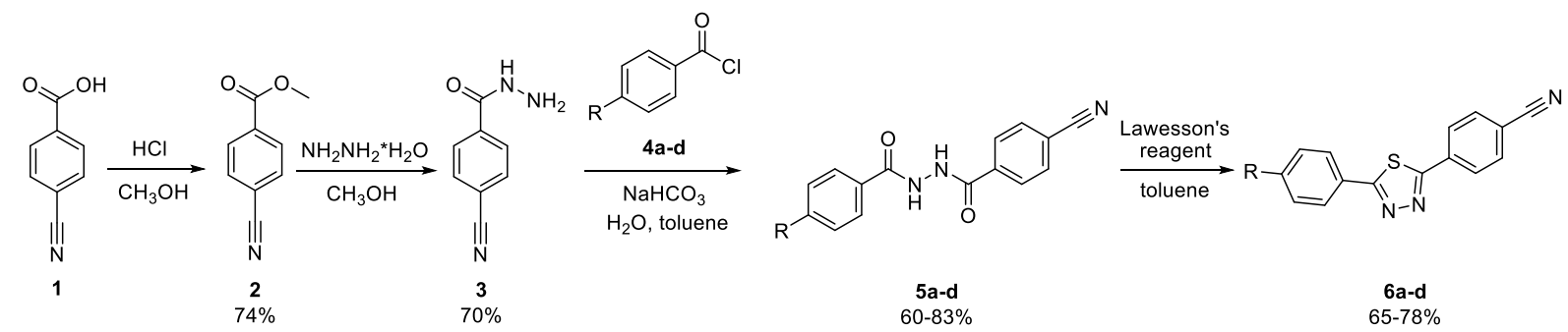

Scheme 1. Synthesis of nitriles containing a 1,3,4-thiadiazole ring $\left[\mathrm{R}=\mathrm{H}, \mathrm{OCH}_{3}, \mathrm{C}\left(\mathrm{CH}_{3}\right)_{3}, \mathrm{NO}_{2}\right]$.

Table 1. Diacylhydrazides (5a-d) and 4-(5-phenyl-1,3,4-thiadiazol-2-yl)benzonitrile derivatives (6a-d)

\begin{tabular}{cccccccc}
\hline \multirow{2}{*}{ Entry } & \multicolumn{5}{c}{ Product 5 } & \multicolumn{4}{c}{ Product 6 } \\
& & Product & Yield (\%) & Product & Reaction time (h) & Yield (\%) \\
\hline 1 & $\mathrm{H}$ & $\mathbf{5 a}$ & 75 & $\mathbf{6 a}$ & 10 & 70 \\
2 & $\mathrm{OCH}_{3}$ & $\mathbf{5 b}$ & 83 & $\mathbf{6 b}$ & 7 & 78 \\
3 & $\mathrm{C}\left(\mathrm{CH}_{3}\right)_{3}$ & $\mathbf{5 c}$ & 80 & $\mathbf{6 c}$ & 9,5 & 74 \\
4 & $\mathrm{NO}_{2}$ & $\mathbf{5 d}$ & 60 & $\mathbf{6 d}$ & 12 & 65 \\
\hline
\end{tabular}

The next step involved the Pinner reaction of previously prepared 4-(5-phenyl-1,3,4-thiadiazol-2yl)benzonitrile derivatives 6a-d with hydrazine hydrate in the presence of sulphur (Scheme 2). Initially, the research involved the formation of symmetrical compounds 7a-d. In this case, there was also a clear influence of the type of substituents. Similar to the previous reaction, electron-donor groups had a very beneficial effect on the yield of the synthesized products. This impact was also visible later in the study which involved obtaining unsymmetrical derivatives 7e-j. The last step was the oxidation of 1,4-dihydro-1,2,4,5-tetrazine derivatives $(\mathbf{7} a-j)$ to the final products. Of the various oxidising agents described in the literature, we decided to use hydrogen peroxide since this environmentally friendly reagent worked well for similar systems containing 1,3,4-oxadiazole. ${ }^{26}$ After 24 hours of stirring at room temperature, a number of the title products 8a-j were isolated with satisfactory yields (Table 2). Traces of two symmetrical products were also detected. The impact of the type of substituents on the outcome of the reaction, and its detailed mechanism, were discussed in our previous work. ${ }^{26}$ 


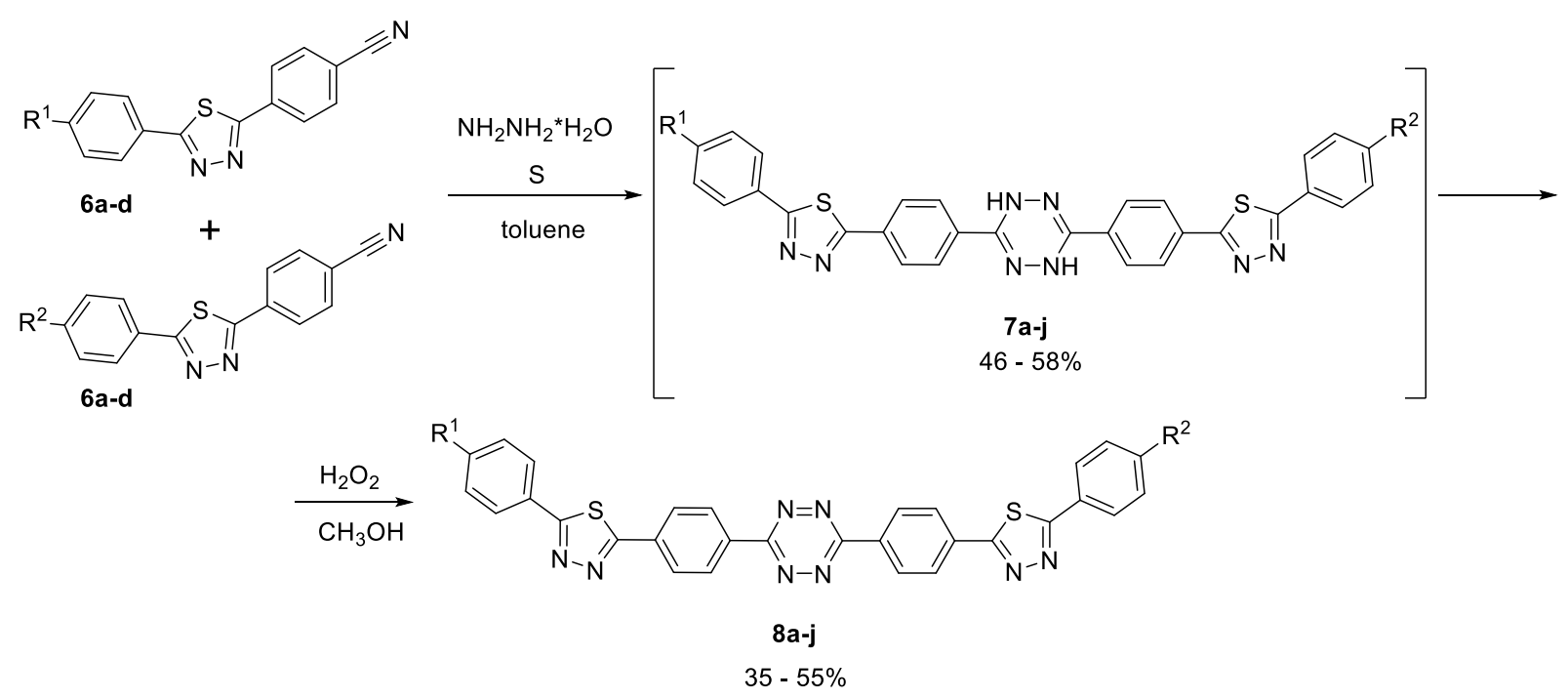

Scheme 2. Synthesis of the final products $\left[\mathrm{R}=\mathrm{H}, \mathrm{OCH}_{3}, \mathrm{C}\left(\mathrm{CH}_{3}\right)_{3}, \mathrm{NO}_{2}\right]$.

Table 2. The resulting symmetrical and unsymmetrical derivatives of $s$-tetrazine conjugated via a 1,4phenylene linker with a 1,3,4-thiadiazole ring (8a-j)

\begin{tabular}{|c|c|c|c|c|c|c|}
\hline \multirow{2}{*}{ Entry } & \multirow{2}{*}{$\mathrm{R}^{1}$} & \multirow{2}{*}{$\mathrm{R}^{2}$} & \multicolumn{2}{|c|}{ Product 7} & \multicolumn{2}{|c|}{ Product 8} \\
\hline & & & Product & Yield (\%) & Product & Yield (\%) \\
\hline 1 & $\mathrm{H}$ & $\mathrm{H}$ & $7 a$ & 70 & $8 a$ & 51 \\
\hline 2 & $\mathrm{OCH}_{3}$ & $\mathrm{OCH}_{3}$ & $7 b$ & 78 & $8 b$ & 55 \\
\hline 3 & $\mathrm{C}\left(\mathrm{CH}_{3}\right)_{3}$ & $\mathrm{C}\left(\mathrm{CH}_{3}\right)_{3}$ & 7c & 65 & $8 c$ & 53 \\
\hline 4 & $\mathrm{NO}_{2}$ & $\mathrm{NO}_{2}$ & $7 d$ & 46 & $8 d$ & 35 \\
\hline 5 & $\mathrm{H}$ & $\mathrm{OCH}_{3}$ & $7 e$ & 54 & $8 e$ & 53 \\
\hline 6 & $\mathrm{H}$ & $\mathrm{C}\left(\mathrm{CH}_{3}\right)_{3}$ & $7 f$ & 48 & $8 f$ & 45 \\
\hline 7 & $\mathrm{H}$ & $\mathrm{NO}_{2}$ & $7 g$ & 44 & $8 g$ & 42 \\
\hline 8 & $\mathrm{OCH}_{3}$ & $\mathrm{C}\left(\mathrm{CH}_{3}\right)_{3}$ & $7 \mathrm{~h}$ & 63 & $8 h$ & 60 \\
\hline 9 & $\mathrm{OCH}_{3}$ & $\mathrm{NO}_{2}$ & $7 \mathbf{i}$ & 45 & $8 i$ & 44 \\
\hline 10 & $\mathrm{C}\left(\mathrm{CH}_{3}\right)_{3}$ & $\mathrm{NO}_{2}$ & $7 \mathbf{j}$ & 45 & $8 j$ & 42 \\
\hline
\end{tabular}

\section{Luminescent properties}

UV-Vis absorption and three-dimensional fluorescence spectra were determined for the final products of the performed reactions (Figure S29-S31, Supplementary Material). In the case of compounds $\mathbf{8 a}, \mathbf{8 c}$, $\mathbf{8 f}$, and $\mathbf{8 g}$, the fluorescence spectra possess one slightly deformed maximum of fluorescence. The global emission maximum of $\mathbf{8 b}, \mathbf{8} \mathbf{d}, \mathbf{8 e}, \mathbf{8 h}, \mathbf{8} \mathbf{i}$, and $\mathbf{8 j}$ covers the second, weaker local maximum (visible as a shoulder of the symmetrical global maximum). Due to large differences in intensities of global and local maxima and a substantial overlap, the $\lambda_{\text {ex }}$ and $\lambda_{\text {em }}$ of local maxima cannot be determined unambiguously, even with the usage of deconvolution of the three-dimensional function $I=f\left(\lambda_{\mathrm{ex}}, \lambda_{\mathrm{em}}\right)$. The described compounds emit fluorescent UV (compounds $\mathbf{8 a}, \mathbf{8 c}, \mathbf{8 f}, \mathbf{8 g}$ ) or Vis (compounds $\mathbf{8 b}, \mathbf{8 d}, \mathbf{8 h}, \mathbf{8 i}, \mathbf{8 j}$ ) radiation upon UV irradiation (Table 3). This suggests that the presence of one terminal $H$ substituent $\left(R^{1}\right.$ or $\left.R^{2}\right)$ is sufficient for appearance of the UV fluorescence. The quantum yields $(\Phi)$ correlate with fluorescence intensities (I), an increase of $\Phi$ causes an increase of I (Figure S32, Supplementary Material), whereas they do not correlate with the 
absorption wavelength (Figure S33, Supplementary Material). This indicates that the electronic transition character is analogous in all the studied compounds, but the amount of the absorbed energy converted into internal energy differs considerably. The main source of excited states is the $n \rightarrow \pi^{*}$ absorption transitions. In general, the fluorescence intensity agrees with the nature of the electron-withdrawing (EWG) and electrondonating (EDG) groups of substituents. The presence of two strong EWG substituents $\left(8 d, R^{1}, R^{2}=N O_{2}\right)$ decreases fluorescence due to a decrease in electron density in delocalised systems. Alternatively, the existence of EDG substituents mostly increases fluorescence. The differences between $\lambda_{\text {em }}$ and $\lambda_{\text {ex }}$ at global maxima fall in two ranges: $121-126 \mathrm{~nm}$ for compounds with the $\mathrm{OCH}_{3}$ substituent $(\mathbf{8 b}, \mathbf{8 e}, \mathbf{8 h}, \mathbf{8 i})$, and $72-91$ $\mathrm{nm}$ for compounds without the $\mathrm{OCH}_{3}$ substituent $(\mathbf{8 a}, \mathbf{8 c}, \mathbf{8 d}, \mathbf{8 f}, \mathbf{8 g}, \mathbf{8 j}$ ). This proves that the studied compounds have diverse energy gaps between individual orbitals participating in fluorescence transitions. It should be noted that compounds with the $\mathrm{OCH}_{3}$ substituent $(\mathbf{8 b}, \mathbf{8 e}, \mathbf{8 h}, \mathbf{8 i})$ possess uncommon fluorescence characteristics as $\lambda_{\mathrm{em}}-\lambda_{\mathrm{ex}}$ is larger than the standard border value of $100 \mathrm{~nm}$. Within each of the abovementioned groups of compounds (with or without the $\mathrm{OCH}_{3}$ substituent), $\lambda_{\text {ex }}$ correlates with $\lambda_{\text {em, }}$ i.e., a larger $\lambda_{\text {ex }}$ produces emission at a larger $\lambda_{\text {em }}$ (Figure S34, Supplementary Material). Noteworthy is the fact that irradiation of compounds $\mathbf{8} \mathbf{h}$ by ultraviolet radiation leads to the emission of violet fluorescence light visible by the naked eye.

Table 3. Luminescence properties of symmetrical (8a-d) and unsymmetrical (8e-j) derivatives of s-tetrazine. Quantum yields $\Phi$ were calculated according to Equation 1 presented in reference ${ }^{27}$, with the use of emission intensity registered for two standard substances: quinine sulphate (qn- $\left.\mathrm{SO}_{4}{ }^{2-}\right)^{28}$ and trans, trans-1,4-diphenyl1,3-butadiene $(\mathrm{dpb}){ }^{29}$ Stokes shifts were calculated from the equation $\Delta=\lambda_{\mathrm{em}}-\lambda_{\text {abs }}$

\begin{tabular}{cccccccc}
\hline Entry & Product & $\begin{array}{c}\lambda_{\text {abs }}(\mathrm{nm}) \\
\text { directly } \\
\text { preceding } \\
\lambda_{\text {em }}\end{array}$ & $\begin{array}{c}\lambda_{\text {ex }}(\mathrm{nm}) \text { at } \\
\text { global } \\
\text { emission } \\
\text { maximum }\end{array}$ & $\begin{array}{c}\lambda_{\text {em }}(\mathrm{nm}) \text { at } \\
\text { global } \\
\text { emission } \\
\text { maximum }\end{array}$ & $\begin{array}{c}\text { Stokes } \\
\text { shift } \\
\Delta[\mathrm{nm}]\end{array}$ & $\begin{array}{c}\Phi_{\mathrm{q}} \\
\left(\mathrm{qn}^{2} \mathrm{SO}_{4}{ }^{2-}\right)\end{array}$ & $\begin{array}{c}\Phi_{\mathrm{d}} \\
(\mathrm{dpb})\end{array}$ \\
\hline 1 & $\mathbf{8 a}$ & 311 & 322 & 399 & 88 & 0.09 & 0.09 \\
2 & $\mathbf{8 b}$ & 306 & 316 & 432 & 126 & 0.39 & 0.39 \\
3 & $\mathbf{8 c}$ & 296 & 304 & 379 & 83 & 0.43 & 0.42 \\
4 & $\mathbf{8 d}$ & 319 & 336 & 410 & 91 & 0.09 & 0.09 \\
5 & $\mathbf{8 e}$ & 303 & 313 & 429 & 126 & 0.41 & 0.40 \\
6 & $\mathbf{8 f}$ & 295 & 300 & 367 & 72 & 0.51 & 0.50 \\
7 & $\mathbf{8 g}$ & 293 & 303 & 367 & 74 & 0.57 & 0.56 \\
8 & $\mathbf{8 h}$ & 323 & 326 & 445 & 122 & 0.54 & 0.53 \\
9 & $\mathbf{8 i}$ & 312 & 318 & 433 & 121 & 0.39 & 0.38 \\
10 & $\mathbf{8 j}$ & 337 & 338 & 411 & 74 & 0.05 & 0.05 \\
\hline
\end{tabular}

The studied compounds (C, Table 4) have generally lower $\Phi$ than their analogous compounds containing 1,3,4oxadiazole $(B)^{26}$ which is a consequence of the sulphur, the presence of which typically causes the severe quenching of fluorescence. ${ }^{30}$ On the other hand, the compounds of both mentioned groups (B and C) exhibit lower $\Phi$ in comparison to their directly conjugated counterparts (A) ${ }^{25}$ It shows that the insertion of benzene rings between tetrazine and thia/oxadiazole rings leads to the disruption of the fluorescence-favourable conjugation. The absorption wavelengths of the title compounds $(\mathbf{C})$ are higher than for the direct-coupled systems (A), and most often are comparable to the corresponding 1,3,4-oxadiazole arrangements (B). The 
above described trend of increasing Stokes shifts for the studied compounds containing a methoxy group is in line with the observations for 1,3,4-oxadiazole analogues, but does not occur in the case of directly conjugated heterocyclic rings (entries 2 and 6, Table 4).

Table 4. Comparison of the properties of the title compounds $(\mathbf{C})$ with analogous systems described in the literature $(\mathbf{A}, \mathbf{B})^{25,26}$

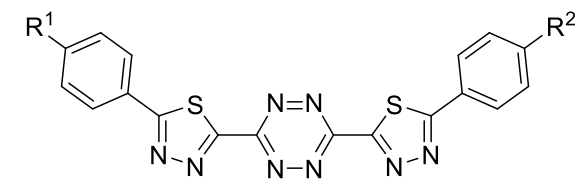

A

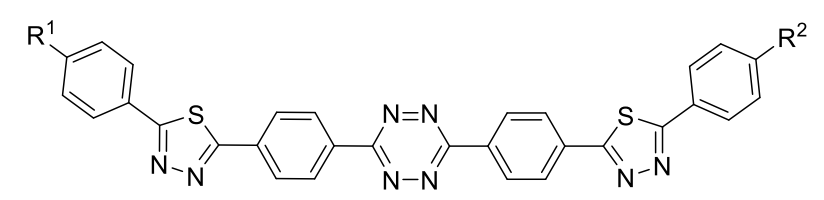

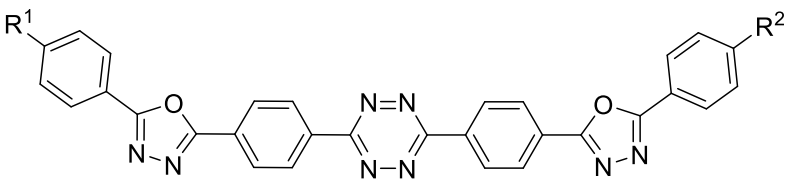

B

C

\begin{tabular}{|c|c|c|c|c|c|c|c|c|c|c|c|}
\hline \multirow[t]{2}{*}{ Entry } & \multirow[t]{2}{*}{$\mathrm{R}^{1}$} & \multirow[t]{2}{*}{$R^{2}$} & \multicolumn{3}{|c|}{ Quantum yield $\Phi$} & \multicolumn{3}{|c|}{$\begin{array}{c}\lambda_{\mathrm{abs}}(\mathrm{nm}) \\
\text { directly preceding } \lambda_{\mathrm{em}}\end{array}$} & \multicolumn{3}{|c|}{ Stokes shift $\Delta[\mathrm{nm}]$} \\
\hline & & & A & B & C & A & B & C & A & B & $\mathbf{C}$ \\
\hline 1 & $\mathrm{H}$ & $\mathrm{H}$ & 0.78 & 0.46 & 0.09 & 265 & 284 & 311 & 85 & 64 & 88 \\
\hline 2 & $\mathrm{OCH}_{3}$ & $\mathrm{OCH}_{3}$ & $>0.98$ & 0.60 & 0.39 & 273 & 309 & 306 & 90 & 129 & 126 \\
\hline 3 & $\mathrm{NO}_{2}$ & $\mathrm{NO}_{2}$ & 0.53 & 0.14 & 0.09 & 274 & 338 & 319 & 91 & 69 & 91 \\
\hline 4 & $\mathrm{H}$ & $\mathrm{NO}_{2}$ & - & 0.26 & 0.57 & - & 298 & 293 & - & 72 & 74 \\
\hline 5 & $\mathrm{C}\left(\mathrm{CH}_{3}\right)_{3}$ & $\mathrm{NO}_{2}$ & - & 0.26 & 0.05 & - & 300 & 337 & - & 84 & 74 \\
\hline 6 & $\mathrm{OCH}_{3}$ & $\mathrm{NO}_{2}$ & - & 0.38 & 0.39 & - & 306 & 312 & - & 129 & 121 \\
\hline
\end{tabular}

\section{Conclusions}

In summary, we have elaborated an efficient and universal methodology for the synthesis of 1,2,4,5-tetrazine derivatives conjugated via a 1,4-phenylene linker with a 1,3,4-thiadiazole ring. The developed procedure made it possible to obtain a number of symmetrical and unsymmetrical derivatives. In addition, the methodology can be used for arrangements containing both electron-donating and electron-withdrawing groups. As a result of the research, ten new final products, not previously described in the literature, were obtained and characterised, including by their fluorescent properties.

\section{Experimental Section}

General. Melting points were measured on a Stuart SMP3 melting point apparatus. NMR spectra were recorded at $25^{\circ} \mathrm{C}$ on an Agilent $400-N M R$ spectrometer at $400 \mathrm{MHz}$ for ${ }^{1} \mathrm{H}$ and $100 \mathrm{MHz}$ for ${ }^{13} \mathrm{C}$, using $\mathrm{CDCl}_{3}$ or DMSO-d6 as solvent and TMS as the internal standard. UV-Vis absorption and 3D fluorescence spectra were registered in methanol solutions $\left(c=5 \cdot 10^{-6} \mathrm{~mol} / \mathrm{dm}^{3}\right)$ with Jasco V-660 and Jasco F-6300 spectrometers, 
respectively. FT-IR spectra were measured between 4000 and $650 \mathrm{~cm}^{-1}$ on an FT-IR Nicolet 6700 apparatus with a Smart iTR accessory. Elemental analyses were performed with a VarioEL analyser. High-resolution mass spectra were obtained by means of a Waters ACQUITY UPLC/Xevo G2QT instrument. Thin-layer chromatography was performed on silica gel $60 \mathrm{~F}_{254}$ (Merck) thin-layer chromatography plates using benzene/ethyl acetate $(1: 3 \mathrm{v} / \mathrm{v})$ or chloroform/ethyl acetate $(5: 1 \mathrm{v} / \mathrm{v})$ as the mobile phases.

Materials and procedures. All chemicals, materials and solvents were purchased from Sigma-Aldrich and used as received. Compounds 1-5 were synthesized according to the literature. ${ }^{26}$

4-(5-Phenyl-1,3,4-thiadiazol-2-yl)benzonitrile derivatives (6a-d). N'-Benzoyl-4-cyanobenzohydrazide (5a) or derivative (5b-d) $(0.02 \mathrm{~mol})$ and Lawesson's reagent $(0.42 \mathrm{~g}, 0.01 \mathrm{~mol})$ were dissolved in toluene $(40 \mathrm{~mL})$ and heated in an oil bath with stirring for 7-12 hours. The resulting mixture was filtered and the residue was evaporated. The crude product was purified by column chromatography using a chloroform/ethyl acetate mixture $(5: 1, v / v)$ as the eluent.

4-(5-Phenyl-1,3,4-thiadiazol-2-yl)benzonitrile (6a). Yellow solid (3.68 g, 70\% yield); mp 197-199 ${ }^{\circ} \mathrm{C} ;{ }^{1} \mathrm{H} \mathrm{NMR}$ $\left(400 \mathrm{MHz}, \mathrm{CDCl}_{3}\right): \delta 7.50-7.53(\mathrm{~m}, 3 \mathrm{H}), 7.79(\mathrm{~d}, J 8.0 \mathrm{~Hz}, 2 \mathrm{H}), 8.00-8.03(\mathrm{~m}, 2 \mathrm{H}), 8.13(\mathrm{~d}, J 8.0 \mathrm{~Hz}, 2 \mathrm{H}) ;{ }^{13} \mathrm{C} \mathrm{NMR}$ $\left(100 \mathrm{MHz}_{2} \mathrm{CDCl}_{3}\right): \delta 114.7,118.2,128.3,128.5,129.5,129.8,131.9,133.1,134.2,166.2,169.7 ;$ UV-VIS: $\lambda_{\max }$ $(\mathrm{MeOH}) 309.0 \mathrm{~nm}\left(\varepsilon \cdot 10^{-3}=11.06 \mathrm{~cm}^{-1} \mathrm{M}^{-1}\right), \lambda_{\max }(\mathrm{MeOH}) 226.0 \mathrm{~nm}\left(\varepsilon \cdot 10^{-3} 12.95 \mathrm{~cm}^{-1} \mathrm{M}^{-1}\right), \lambda_{\max }(\mathrm{MeOH}) 204.0$ $\mathrm{nm}\left(\varepsilon \cdot 10^{-3}=17.12 \mathrm{~cm}^{-1} \mathrm{M}^{-1}\right)$; IR (ATR) v:3112, 2839, 2554, 2227, 1669, 1604, 1519, 1492, 1423, 1344, 1309, 1292, 1178, 1128, 1105, 1070, 1013, 965, 930, 877, 853, 800, 788, 772, 739, 716, $691 \mathrm{~cm}^{-1}$; Anal. Calcd for $\mathrm{C}_{15} \mathrm{H}_{9} \mathrm{~N}_{3} \mathrm{~S}$ : C, 68.42; $\mathrm{H}, 3.45 ; \mathrm{N}, 15.96$. Found: $\mathrm{C}, 68.44 ; \mathrm{H}, 3.42 ; \mathrm{N}, 15.95 ; \mathrm{HRMS}$ calcd for $\left(\mathrm{C}_{15} \mathrm{H}_{9} \mathrm{~N}_{3} \mathrm{~S}+\mathrm{H}^{+}\right)$: 264.0590; found: 264.0588 .

4-(5-(4-Methoxyphenyl)-1,3,4-thiadiazol-2-yl)benzonitrile (6b). Yellow solid (4.57 g, 78\% yield); mp 196-198 ${ }^{\circ} \mathrm{C} ;{ }^{1} \mathrm{H}$ NMR (400 MHz, DMSO-d 6 ): $\delta 3.87(\mathrm{~s}, 3 \mathrm{H}), 7.00$ (d, J $\left.4.0 \mathrm{~Hz}, 2 \mathrm{H}\right), 7.17(\mathrm{~d}, J 8.0 \mathrm{~Hz}, 2 \mathrm{H}), 8.09(\mathrm{~d}, J 8.0 \mathrm{~Hz}$, $2 \mathrm{H}), 8.28(\mathrm{~d}, J 8.0 \mathrm{~Hz}, 2 \mathrm{H}) ;{ }^{13} \mathrm{C}$ NMR $\left(100 \mathrm{MHz}, \mathrm{DMSO}-d_{6}\right): \delta 55.4,113.4,114.9,118.3,127.2,128.2,128.8$, 129.5, 133.3, 161.1, 162.3, 164.6; UV-VIS: $\lambda_{\max }(\mathrm{MeOH}) 312.0 \mathrm{~nm}\left(\varepsilon \cdot 10^{-3} 8.66 \mathrm{~cm}^{-1} \mathrm{M}^{-1}\right), \lambda_{\max }(\mathrm{MeOH}) 228.5 \mathrm{~nm}$ $\left(\varepsilon \cdot 10^{-3} 13.48 \mathrm{~cm}^{-1} \mathrm{M}^{-1}\right), \lambda_{\max }(\mathrm{MeOH}) 204.0 \mathrm{~nm}\left(\varepsilon \cdot 10^{-3}=17.65 \mathrm{~cm}^{-1} \mathrm{M}^{-1}\right) ;$ IR (ATR) v: 3063, 2964, 2841, 2552, 2228, 1687, 1602, 1550, 1519, 1492, 1434, 1407, 1345, 1309, 1295, 1258, 1172, 1130, 1105, 1073, 1015, 987, 966, 936, 854, 843, 830, 800, 772, 740, $712 \mathrm{~cm}^{-1}$; Anal. Calcd for $\mathrm{C}_{16} \mathrm{H}_{11} \mathrm{~N}_{3} \mathrm{OS}: \mathrm{C}, 65.51 ; \mathrm{H}, 3.78 ; \mathrm{N}, 14.32$. Found: $\mathrm{C}, 65.50 ; \mathrm{H}, 3.76 ; \mathrm{N}, 14.34$; HRMS calcd for $\left(\mathrm{C}_{16} \mathrm{H}_{11} \mathrm{~N}_{3} \mathrm{OS}+\mathrm{H}^{+}\right)$: 294.0696; found: 294.0694.

4-(5-(4-(tert-Butyl)phenyl)-1,3,4-thiadiazol-2-yl)benzonitrile (6c). Yellow solid (4.72 g, 74\% yield); mp 168-170 ${ }^{\circ} \mathrm{C} ;{ }^{1} \mathrm{H}$ NMR $\left(400 \mathrm{MHz}, \mathrm{DMSO}-d_{6}\right): \delta 1.34(\mathrm{~s}, 9 \mathrm{H}), 7.51(\mathrm{~d}, J 8.0 \mathrm{~Hz}, 2 \mathrm{H}), 7.88(\mathrm{~d}, J 8.0 \mathrm{~Hz}, 2 \mathrm{H}), 8.03-8.10(\mathrm{~m}, 4 \mathrm{H})$; ${ }^{13} \mathrm{C}$ NMR $\left(100 \mathrm{MHz}\right.$, DMSO- $\left.d_{6}\right): \delta 30.8,34.7,113.8,120.5,126.2,126.6,127.3,128.2,129.4,132.6,155.8$, 163.3, 167.2; UV-VIS: $\lambda_{\max }(\mathrm{MeOH}) 230.0 \mathrm{~nm}\left(\varepsilon \cdot 10^{-3}=19.47 \mathrm{~cm}^{-1} \mathrm{M}^{-1}\right), \lambda_{\max }(\mathrm{MeOH}) 203.5 \mathrm{~nm}\left(\varepsilon \cdot 10^{-3}=19.66 \mathrm{~cm}\right.$ ${ }^{1} \mathrm{M}^{-1}$ ); IR (ATR) v: 3220, 2953, 1695, 1671, 1634, 1600, 1572, 1507, 1459, 1439, 1303, 1281, 1255, 1169, 1123, 1015, 989, 917, 852, 838, 824, 800, $704 \mathrm{~cm}^{-1}$; Anal. Calcd for $\mathrm{C}_{19} \mathrm{H}_{17} \mathrm{~N}_{3} \mathrm{~S}: \mathrm{C}, 71.44 ; \mathrm{H}, 5.36 ; \mathrm{N}, 13.16$. Found: C, 71.42; $\mathrm{H}, 5.37 ; \mathrm{N}, 13.18$; HRMS calcd for $\left(\mathrm{C}_{19} \mathrm{H}_{17} \mathrm{~N}_{3} \mathrm{~S}+\mathrm{H}^{+}\right)$: 320.1216; found: 320.1218 .

4-(5-(4-Nitrophenyl)-1,3,4-thiadiazol-2-yl)benzonitrile (6d). Yellow solid (4.00 g, 65\% yield); mp $226-228{ }^{\circ} \mathrm{C}$; ${ }^{1} \mathrm{H}$ NMR (400 MHz, DMSO- $\left.d_{6}\right): \delta 8.12-8.18(\mathrm{~m}, 2 \mathrm{H}), 8.31-8.36(\mathrm{~m}, 2 \mathrm{H}), 8.42(\mathrm{~d}, J 8 \mathrm{~Hz}, 2 \mathrm{H}), 8.47(\mathrm{~d}, J 8 \mathrm{~Hz}, 2 \mathrm{H})$; ${ }^{13} \mathrm{C}$ NMR $\left(100 \mathrm{MHz}\right.$, DMSO- $\left.d_{6}\right): \delta 113.4,114.4,118.0,127.6,128.2,129.0,130.7,132.3,150.0,161.1,166.8$; UV-VIS: $\lambda_{\max }(\mathrm{MeOH}) 296.5 \mathrm{~nm}\left(\varepsilon \cdot 10^{-3}=1.97 \mathrm{~cm}^{-1} \mathrm{M}^{-1}\right), \lambda_{\max }(\mathrm{MeOH}) 225.5 \mathrm{~nm}\left(\varepsilon \cdot 10^{-3}=3.94 \mathrm{~cm}^{-1} \mathrm{M}^{-1}\right), \lambda_{\max }$ $(\mathrm{MeOH}) 204.0 \mathrm{~nm}\left(\varepsilon \cdot 10^{-3}=5.66 \mathrm{~cm}^{-1} \mathrm{M}^{-1}\right)$; IR (ATR) v: 2953, 2233, 2168, 1689, 1635, 1600, 1571, 1549, 1523, 1507, 1460, 1439, 1348, 1283, 1256, 1196, 1177, 1141, 1108, 1014, 991, 927, 879, 853, 837, 800, 752, 738, $709 \mathrm{~cm}^{-1}$; Anal. Calcd for $\mathrm{C}_{15} \mathrm{H}_{8} \mathrm{~N}_{4} \mathrm{O}_{2} \mathrm{~S}$ : C, 58.44; $\mathrm{H}, 2.62 ; \mathrm{N}, 18.17$. Found: $\mathrm{C}, 58.45 ; \mathrm{H}, 2.60 ; \mathrm{N}, 18.16$; HRMS calcd for $\left(\mathrm{C}_{15} \mathrm{H}_{8} \mathrm{~N}_{4} \mathrm{O}_{2} \mathrm{~S}+\mathrm{H}^{+}\right)$: 309.0441; found: 309.0443 . 
Preparation of final products (8a-j). The mixtures of one or two of compounds $6 a-d(2 \mathrm{mmol}$ of each substrate), sulphur $(0.08 \mathrm{~g}, 2.5 \mathrm{mmol})$ and dry toluene $(60 \mathrm{~mL})$ was cooled to $0{ }^{\circ} \mathrm{C}$ and hydrazine hydrate $(0.5$ $\mathrm{mL}, 6 \mathrm{mmol}$ ) was added dropwise with stirring. The slurry was allowed to reach room temperature and then heated under reflux for $2 \mathrm{~h}$. The mixture was then cooled to room temperature, filtered, and the filtrate was evaporated. The crude product (7a-j) was dissolved in methanol $(30 \mathrm{~mL})$, and then $35 \%$ hydrogen peroxide (30 $\mathrm{mL}$ ) was added. After stirring at room temperature for $24 \mathrm{~h}$, the reaction mixture was filtered and concentrated by evaporation. The oily residue or precipitate was filtered and purified by column chromatography using a chloroform/ethyl acetate mixture $(5: 1, v / v)$ as the eluent.

3,6-Bis(4-(5-phenyl-1,3,4-thiadiazol-2-yl)phenyl)-1,4-dihydro-1,2,4,5-tetrazine (7a). Yellow solid (1.55 g, 70\% yield).

3,6-Bis(4-(5-(4-methoxyphenyl)-1,3,4-thiadiazol-2-yl)phenyl)-1,4-dihydro-1,2,4,5-tetrazine (7b). Yellow solid (1.91 g, 78\% yield).

3,6-Bis(4-(5-(4-(tert-butyl)phenyl)-1,3,4-thiadiazol-2-yl)phenyl)-1,4-dihydro-1,2,4,5-tetrazine (7c). Yellow solid (1.73 g, 65\% yield).

3,6-Bis(4-(5-(4-nitrophenyl)-1,3,4-thiadiazol-2-yl)phenyl)-1,4-dihydro-1,2,4,5-tetrazine (7d). Yellow solid (1.18 g, 46\% yield).

2-(4-Methoxyphenyl)-5-(4-(6-(4-(5-phenyl-1,3,4-thiadiazol-2-yl)phenyl)-1,4-dihydro-1,2,4,5-tetrazin-3yl)phenyl)-1,3,4-thiadiazole (7e). Yellow solid (1.26 g, 54\% yield).

2-(4-(tert-Butyl)phenyl)-5-(4-(6-(4-(5-phenyl-1,3,4-thiadiazol-2-yl)phenyl)-1,4-dihydro-1,2,4,5-tetrazin-3yl)phenyl)-1,3,4-thiadiazole (7f). Yellow solid (1.17 g, 48\% yield).

2-(4-Nitrophenyl)-5-(4-(6-(4-(5-phenyl-1,3,4-thiadiazol-2-yl)phenyl)-1,4-dihydro-1,2,4,5-tetrazin-3yl)phenyl)-1,3,4-thiadiazole (7g). Yellow solid (1.05 g, 44\% yield).

2-(4-(tert-Butyl)phenyl)-5-(4-(6-(4-(5-(4-methoxyphenyl)-1,3,4-thiadiazol-2-yl)phenyl)-1,4-dihydro-1,2,4,5tetrazin-3-yl)phenyl)-1,3,4-thiadiazole (7h). Yellow solid (1.61 g, 63\% yield).

2-(4-Methoxyphenyl)-5-(4-(6-(4-(5-(4-nitrophenyl)-1,3,4-thiadiazol-2-yl)phenyl)-1,4-dihydro-1,2,4,5-tetrazin3-yl)phenyl)-1,3,4-thiadiazole (7i). Yellow solid (1.13 g, 45\% yield).

2-(4-(tert-Butyl)phenyl)-5-(4-(6-(4-(5-(4-nitrophenyl)-1,3,4-thiadiazol-2-yl)phenyl)-1,4-dihydro-1,2,4,5tetrazin-3-yl)phenyl)-1,3,4-thiadiazole(7j). Yellow solid (1.18 g, 45\% yield).

3,6-Bis(4-(5-phenyl-1,3,4-thiadiazol-2-yl)phenyl)-1,2,4,5-tetrazine (8a). Yellow solid (0.79 g, 51\% yield); mp 218-220 ${ }^{\circ} \mathrm{C} ;{ }^{1} \mathrm{H}$ NMR $\left(400 \mathrm{MHz}, \mathrm{CDCl}_{3}\right): \delta 7.47-7.50(\mathrm{~m}, 6 \mathrm{H}), 7.76(\mathrm{~d}, \mathrm{~J} 8.0 \mathrm{~Hz}, 4 \mathrm{H}), 7.96-7.99(\mathrm{~m}, 4 \mathrm{H}), 8.09(\mathrm{~d}, J$ $8.0 \mathrm{~Hz}, 4 \mathrm{H}) ;{ }^{13} \mathrm{C} \mathrm{NMR}\left(100 \mathrm{MHz}, \mathrm{CDCl}_{3}\right): \delta 127.2,127.4,128.4,129.3,129.4131 .2,131.7,133.0,166.0,168.2$, 169.5; UV-VIS: $\lambda_{\max }(\mathrm{MeOH}) 311 \mathrm{~nm}\left(\varepsilon \cdot 10^{-3}=50.0 \mathrm{~cm}^{-1} \mathrm{M}^{-1}\right)$; fluorescence $\left(\mathrm{CH}_{3} \mathrm{OH}\right): \lambda_{\mathrm{ex}}=322 \mathrm{~nm} ; \lambda_{\mathrm{em}}=399 \mathrm{~nm}$; IR (ATR) v: 3375, 2229, 1675, 1608, 1552, 1519, 1493, 1441, 1413, 1307, 1253, 1174, 1100, 1070, 1026, 988, $962,832,770,743,703 \mathrm{~cm}^{-1}$; Anal. Calcd for $\mathrm{C}_{30} \mathrm{H}_{18} \mathrm{~N}_{8} \mathrm{~S}_{2}: \mathrm{C}, 64.97 ; \mathrm{H}, 3.27 ; \mathrm{N}, 20.20$. Found: C, 64.99; $\mathrm{H}, 3.25$; $\mathrm{N}, 20.21$; HRMS calcd for $\left(\mathrm{C}_{30} \mathrm{H}_{18} \mathrm{~N}_{8} \mathrm{~S}_{2}+\mathrm{H}^{+}\right)$: 555.1169; found: 555.1168.

3,6-Bis(4-(5-(4-methoxyphenyl)-1,3,4-thiadiazol-2-yl)phenyl)-1,2,4,5-tetrazine (8b). Yellow solid (1.05 g, 55\% yield); mp 169-171 ${ }^{\circ} \mathrm{C} ;{ }^{1} \mathrm{H}$ NMR (400 MHz, DMSO-d 6 ): $\delta 3.87(\mathrm{~s}, 6 \mathrm{H}), 7.01(\mathrm{~d}, J 8.0 \mathrm{~Hz}, 4 \mathrm{H}), 7.17(\mathrm{~d}, J 8.0 \mathrm{~Hz}, 4 \mathrm{H})$, $8.09(\mathrm{~d}, J 8.0 \mathrm{~Hz}, 4 \mathrm{H}), 8.19$ (d, J 8.0 Hz, 4H); ${ }^{13} \mathrm{C}$ NMR $\left(100 \mathrm{MHz}, \mathrm{DMSO}-d_{6}\right): \delta 55.4,114.9,122.9,126.4,128.4$, 128.7, 129.4, 133.3, 162.2, 162.8, 164.2, 167.0; UV-VIS: $\lambda_{\max }(\mathrm{MeOH}) 246 \mathrm{~nm}\left(\varepsilon \cdot 10^{-3}=31.15 \mathrm{~cm}^{-1} \mathrm{M}^{-1}\right)$, $\lambda_{\max }(\mathrm{MeOH}) 306 \mathrm{~nm}\left(\varepsilon \cdot 10^{-3}=39.8 \mathrm{~cm}^{-1} \mathrm{M}^{-1}\right)$; fluorescence $\left(\mathrm{CH}_{3} \mathrm{OH}\right): \lambda_{\mathrm{ex}}=316 \mathrm{~nm} ; \lambda_{\mathrm{em}}=432 \mathrm{~nm} ;$ IR (ATR) v: 2229, 2022, 1994, 1676, 1610, 1582, 1548, 1519, 1492, 1411, 1305, 1252, 1172, 1102, 1072, 1028, 962, 834, 813, 773, 745, 731, 714, 703, $684 \mathrm{~cm}^{-1}$; Anal. Calcd for $\mathrm{C}_{32} \mathrm{H}_{22} \mathrm{~N}_{8} \mathrm{O}_{2} \mathrm{~S}_{2}$ : C, 62.53; $\mathrm{H}, 3.61 ; \mathrm{N}, 18.23$. Found: C, 62.54; $\mathrm{H}, 3.60 ; \mathrm{N}, 28.22$; HRMS calcd for $\left(\mathrm{C}_{32} \mathrm{H}_{22} \mathrm{~N}_{8} \mathrm{O}_{2} \mathrm{~S}_{2}+\mathrm{H}^{+}\right)$: 615.1380; found: 615.1379 . 
3,6-Bis(4-(5-(4-(tert-butyl)phenyl)-1,3,4-thiadiazol-2-yl)phenyl)-1,2,4,5-tetrazine (8c). Yellow solid (0.92 g, 53\% yield); mp $145-147{ }^{\circ} \mathrm{C} ;{ }^{1} \mathrm{H}$ NMR (400 MHz, DMSO-d $)$ : $\delta 1.33(\mathrm{~s}, 18 \mathrm{H}), 7.64(\mathrm{~d}, J 8.0 \mathrm{~Hz}, 4 \mathrm{H}), 7.87$ (d, J 8.0 $\mathrm{Hz}, 4 \mathrm{H}), 8.02-8.10(\mathrm{~m}, 8 \mathrm{H}) ;{ }^{13} \mathrm{C}$ NMR $\left(100 \mathrm{MHz}, \mathrm{DMSO}-d_{6}\right): \delta 30.8,34.8,125.2,126.2,126.5,126.8,127.3$, 128.5, 133.3, 155.3, 162.7, 163.8, 165.7; UV-VIS : $\lambda_{\max }(\mathrm{MeOH}) 241 \mathrm{~nm}\left(\varepsilon \cdot 10^{-3}=28.98 \mathrm{~cm}^{-1} \mathrm{M}^{-1}\right), \lambda_{\max }(\mathrm{MeOH}) 296$ $\mathrm{nm}\left(\varepsilon \cdot 10^{-3}=54.62 \mathrm{~cm}^{-1} \mathrm{M}^{-1}\right)$; fluorescence $\left(\mathrm{CH}_{3} \mathrm{OH}\right): \lambda_{\mathrm{ex}}=304 \mathrm{~nm} ; \lambda_{\mathrm{em}}=379 \mathrm{~nm}$; IR (ATR) v: 2964, 2228, 1672, $1624,1613,1581,1548,1492,1446,1417,1363,1308,1272,1119,1098,1070,1015,842,773,751,735,710$ $\mathrm{cm}^{-1}$; Anal. Calcd for $\mathrm{C}_{38} \mathrm{H}_{34} \mathrm{~N}_{8} \mathrm{~S}_{2}$ : C, 68.44; H, 5.14; N, 16.80. Found: C, 68.42; H, 5.15; N, 16.82; HRMS calcd for $\left(\mathrm{C}_{38} \mathrm{H}_{34} \mathrm{~N}_{8} \mathrm{~S}_{2}+\mathrm{H}^{+}\right): 667.2421$; found: 667.2423 .

3,6-Bis(4-(5-(4-nitrophenyl)-1,3,4-thiadiazol-2-yl)phenyl)-1,2,4,5-tetrazine (8d). Yellow solid (0.41 g, 35\% yield); mp 116-118 ${ }^{\circ} \mathrm{C} ;{ }^{1} \mathrm{H}$ NMR (400 MHz, DMSO-d $): \delta 8.12(\mathrm{~d}, J 8 \mathrm{~Hz}, 4 \mathrm{H}), 8.30-8.35(\mathrm{~m}, 4 \mathrm{H}), 8.40(\mathrm{~d}, J 8 \mathrm{~Hz}$, $4 \mathrm{H}), 8.45$ (d, J $8 \mathrm{~Hz}, 4 \mathrm{H}) ;{ }^{13} \mathrm{C}$ NMR (100 MHz, DMSO): $\delta 116.2,127.1,128.1,128.3,128.4,128.5,129.2,152.2$, 162.1, 164.2, 166.9; UV-VIS: $\lambda_{\max }(\mathrm{MeOH})=319 \mathrm{~nm}\left(\varepsilon \cdot 10^{-3}=36.34 \mathrm{~cm}^{-1} \mathrm{M}^{-1}\right)$; fluorescence $\left(\mathrm{CH}_{3} \mathrm{OH}\right): \lambda_{\mathrm{ex}}=336$ $\mathrm{nm} ; \lambda_{\mathrm{em}}=410 \mathrm{~nm}$; IR (ATR) v: 2228, 2157, 2025, 1698, 1647, 1614, 1595, 1567, 1545, 1525, 1479, 1406, 1344, 1297, 1280, 1253, 1194, 1106, 1073, 1012, 962, 949, 881, 849, 830, 813, 783, 776, 739, 721, 711, 692, 681,668 $\mathrm{cm}^{-1}$; Anal. Calcd for $\mathrm{C}_{30} \mathrm{H}_{16} \mathrm{~N}_{10} \mathrm{O}_{4} \mathrm{~S}_{2}$ : C, 55.90; H, 2.50; N, 21.73. Found: C, 55.91; H, 2.48; N, 21.74; HRMS calcd for $\left(\mathrm{C}_{30} \mathrm{H}_{16} \mathrm{~N}_{10} \mathrm{O}_{4} \mathrm{~S}_{2}+\mathrm{H}^{+}\right)$: 645.0870; found: 645.0872 .

\section{2-(4-Methoxyphenyl)-5-(4-(6-(4-(5-phenyl-1,3,4-thiadiazol-2-yl)phenyl)-1,2,4,5-tetrazin-3-yl)phenyl)-1,3,4-}

thiadiazole (8e). Yellow solid (0.67 g, 53\% yield); mp 231-233 ${ }^{\circ} \mathrm{C} ;{ }^{1} \mathrm{H}$ NMR (400 MHz, DMSO- $\left.d_{6}\right): \delta 3.86(\mathrm{~s}, 3 \mathrm{H})$, $7.13(\mathrm{~d}, J 8.0 \mathrm{~Hz}, 2 \mathrm{H}), 7.16(\mathrm{~d}, J 8.0 \mathrm{~Hz}, 2 \mathrm{H}), 7.63-7.67(\mathrm{~m}, 3 \mathrm{H}), 7.92(\mathrm{~d}, J 12 \mathrm{~Hz}, 2 \mathrm{H}), 8.03(\mathrm{~d}, J 8.0 \mathrm{~Hz}, 2 \mathrm{H}), 8.08$ $(\mathrm{d}, J 8.0 \mathrm{~Hz}, 4 \mathrm{H}), 8.14-8.19(\mathrm{~m}, 2 \mathrm{H}) ;{ }^{13} \mathrm{C}$ NMR $\left(100 \mathrm{MHz}, \mathrm{DMSO}-d_{6}\right): \delta 55.5,114.9,122.1,126.4,126.7,127.2$, 127.4, 128.2, 128.4, 128.6, 128.7, 129.1, 129.4, 129.5, 132.0, 133.3, 161.5, 161.9, 162.3, 163.4, 164.6, 166.5, 168.7; UV-VIS: $\lambda_{\max }(\mathrm{MeOH}) 251 \mathrm{~nm}\left(\varepsilon \cdot 10^{-3}=23.3 \mathrm{~cm}^{-1} \mathrm{M}^{-1}\right), 303 \mathrm{~nm}\left(\varepsilon \cdot 10^{-3}=50.2 \mathrm{~cm}^{-1} \mathrm{M}^{-1}\right)$; fluorescence $\left(\mathrm{CH}_{3} \mathrm{OH}\right): \lambda_{\mathrm{ex}}=313 \mathrm{~nm} ; \lambda_{\mathrm{em}}=429 \mathrm{~nm}$; IR (ATR) v: 3378, 2228, 1671, 1608, 1550, 1519, 1484, 1441, 1414, 1307, $1253,1174,1118,1100,1069,1027,962,832,769,744,704,690,667 \mathrm{~cm}^{-1}$; Anal. Calcd for $\mathrm{C}_{31} \mathrm{H}_{20} \mathrm{~N}_{8} \mathrm{OS}_{2}: \mathrm{C}_{\text {, }}$ 63.68; $\mathrm{H}, 3.45 ; \mathrm{N}, 19.17$. Found: $\mathrm{C}, 63.70 ; \mathrm{H}, 3.46 \mathrm{~N}, 19.16$; HRMS calcd for $\left(\mathrm{C}_{31} \mathrm{H}_{20} \mathrm{~N}_{8} \mathrm{OS}_{2}+\mathrm{H}^{+}\right): 585.1274 ;$ found: 585.1273.

2-(4-(tert-Butyl)phenyl)-5-(4-(6-(4-(5-phenyl-1,3,4-thiadiazol-2-yl)phenyl)-1,2,4,5-tetrazin-3-yl)phenyl)-1,3,4thiadiazole (8f). Yellow solid (0.53 g, 45\% yield); mp $161-163^{\circ} \mathrm{C} ;{ }^{1} \mathrm{H}$ NMR $\left(400 \mathrm{MHz}, \mathrm{DMSO}-d_{6}\right): \delta 1.29(\mathrm{~s}, 9 \mathrm{H})$, 7.61-7.67 (m, 5H), $7.87(\mathrm{~d}, J 8.0 \mathrm{~Hz}, 2 \mathrm{H}), 8.01-8.05(\mathrm{~m}, 2 \mathrm{H}), 8.13(\mathrm{~d}, J 2.4 \mathrm{~Hz}, 4 \mathrm{H}), 8.15(\mathrm{~d}, J 1.6 \mathrm{~Hz}, 4 \mathrm{H}) ;{ }^{13} \mathrm{C} \mathrm{NMR}$ $\left(100 \mathrm{MHz}, \mathrm{DMSO}-d_{6}\right): \delta 30.9,34.6,123.4,125.4,126.7,126.8,127.2,127.3,127.8,128,3,129.2,129.4,129.5$, 131.7, 132.3, 133.3, 133.4, 155.3, 162.9, 164.6, 165.7, 165.9, 166.1, 169.0; UV-VIS: $\lambda_{\max }(\mathrm{MeOH}) 295 \mathrm{~nm}\left(\varepsilon \cdot 10^{-3}\right.$ $\left.=55.42 \mathrm{~cm}^{-1} \mathrm{M}^{-1}\right)$; fluorescence $\left(\mathrm{CH}_{3} \mathrm{OH}\right): \lambda_{\mathrm{ex}}=300 \mathrm{~nm} ; \lambda_{\mathrm{em}}=367 \mathrm{~nm}$; IR (ATR) v: 2228, 1671, 1610, 1572, 1550, $1491,1447,1414,1307,1253,1175,1067,1029,853,833,775,739,708,687 \mathrm{~cm}^{-1}$; Anal. Calcd for $\mathrm{C}_{34} \mathrm{H}_{26} \mathrm{~N}_{8} \mathrm{~S}_{2}$ : C, 66.86; H, 4.29; N, 18.35. Found: C, 66.85; H, 4.27; N, 18.36; HRMS calcd for $\left(\mathrm{C}_{34} \mathrm{H}_{26} \mathrm{~N}_{8} \mathrm{~S}_{2}+\mathrm{H}^{+}\right): 611.1795$; found: 611.1793 .

\section{2-(4-Nitrophenyl)-5-(4-(6-(4-(5-phenyl-1,3,4-thiadiazol-2-yl)phenyl)-1,2,4,5-tetrazin-3-yl)phenyl)-1,3,4-}

thiadiazole $(8 \mathrm{~g})$. Yellow solid (0.44 g, $42 \%$ yield); $\mathrm{mp} 165-167{ }^{\circ} \mathrm{C} ;{ }^{1} \mathrm{H}$ NMR (400 MHz, DMSO- $\left.d_{6}\right): \delta 7.63-7.71(\mathrm{~m}$, 3H), $8.01(\mathrm{~d}, J 8.0 \mathrm{~Hz}, 2 \mathrm{H}), 8.06(\mathrm{~d}, J 8.0 \mathrm{~Hz}, 4 \mathrm{H}), 8.14(\mathrm{~d}, J 8.0 \mathrm{~Hz}, 4 \mathrm{H}), 8.24(\mathrm{~d}, J 8.0 \mathrm{~Hz}, 2 \mathrm{H}), 8.44(\mathrm{~d}, J 8.0 \mathrm{~Hz}$, $2 \mathrm{H}) ;{ }^{13} \mathrm{C}$ NMR $\left(100 \mathrm{MHz}\right.$, DMSO- $\left.d_{6}\right): \delta 118.3,123.4,126.8,127.0,127.3,127.7,128.0,128.3,129.1,129.4$, $129.5,131.7,132.1,133.3,133.4 ; 152.3,162.9,164.6,166.1,166.9,169.0,169.9 ;$ UV-VIS: $\lambda_{\max }(\mathrm{MeOH}) 293 \mathrm{~nm}$ $\left(\varepsilon \cdot 10^{-3}=72.28 \mathrm{~cm}^{-1} \mathrm{M}^{-1}\right)$; fluorescence $\left(\mathrm{CH}_{3} \mathrm{OH}\right): \lambda_{\mathrm{ex}}=303 \mathrm{~nm} ; \lambda_{\mathrm{em}}=367 \mathrm{~nm} ;$ IR (ATR) v: 2228, 1634, 1608, 1576, 1550, 1490, 1446, 1413, 1341, 1307, 1273, 1177, 1121, 1104, 1067, 1031, 853, 775, 739, 708, $687 \mathrm{~cm}^{-1}$; Anal. Calcd for $\mathrm{C}_{30} \mathrm{H}_{17} \mathrm{~N}_{9} \mathrm{O}_{2} \mathrm{~S}_{2}: \mathrm{C}, 60.09 ; \mathrm{H}, 2.86 ; \mathrm{N}, 21.02$. Found: $\mathrm{C}, 60.08 ; \mathrm{H}, 2.88 ; \mathrm{N}, 21.01$; HRMS calcd for $\left(\mathrm{C}_{30} \mathrm{H}_{17} \mathrm{~N}_{9} \mathrm{O}_{2} \mathrm{~S}_{2}+\mathrm{H}^{+}\right)$: 600.1019; found: 600.1017 . 
2-(4-(tert-Butyl)phenyl)-5-(4-(6-(4-(5-(4-methoxyphenyl)-1,3,4-thiadiazol-2-yl)phenyl)-1,2,4,5-tetrazin-3-

yl)phenyl)-1,3,4-thiadiazole (8h). Note: the product is a precipitate formed after the reaction. Yellow solid (0.97 g, 60\% yield); mp 201-203ㄷ; ${ }^{1} \mathrm{H}$ NMR (400 MHz, DMSO-d $): \delta 1.32$ (s, 9H), $3.86(\mathrm{~s}, 3 \mathrm{H}), 7.14(\mathrm{~d}, J 8.0 \mathrm{~Hz}$, 2H), $7.16(\mathrm{~d}, J 8.0 \mathrm{~Hz}, 2 \mathrm{H}), 7.63(\mathrm{~d}, J 4.0 \mathrm{~Hz}, 2 \mathrm{H}), 7.65$ (d, J $4.0 \mathrm{~Hz}, 2 \mathrm{H}), 8.02$ (d, J $8.0 \mathrm{~Hz}, 2 \mathrm{H}), 8.07(\mathrm{~d}, J 8.0 \mathrm{~Hz}$, $4 \mathrm{H}), 8.26(\mathrm{~d}, J 8.0 \mathrm{~Hz}, 2 \mathrm{H}) ;{ }^{13} \mathrm{C}$ NMR $\left(100 \mathrm{MHz}, \mathrm{DMSO}-d_{6}\right): \delta 30.7,34.8,55.5,114.8 ; 121.7,125.2,127.2,127.3$, $127.4,128.2,128.3,128.7,129.1,129.5,129.8,133.3,133.6,154.9,161.9,162.3,162.4,164.6,165.2,165.7$, 166.6; UV-VIS: $\lambda_{\max }(\mathrm{MeOH}) 225 \mathrm{~nm}\left(\varepsilon \cdot 10^{-3}=39.82 \mathrm{~cm}^{-1} \mathrm{M}^{-1}\right), 251 \mathrm{~nm}\left(\varepsilon \cdot 10^{-3}=28.5 \mathrm{~cm}^{-1} \mathrm{M}^{-1}\right), 323 \mathrm{~nm}\left(\varepsilon \cdot 10^{-3}=\right.$ $\left.53.5 \mathrm{~cm}^{-1} \mathrm{M}^{-1}\right)$; fluorescence $\left(\mathrm{CH}_{3} \mathrm{OH}\right): \lambda_{\mathrm{ex}}=326 \mathrm{~nm} ; \lambda_{\mathrm{em}}=445 \mathrm{~nm}$; IR (ATR) v: 2228, 1672, 1634, 1607, 1549, $1519,1436,1407,1306,1256,1172,1122,1101,1073,1024,830,798,769,745,704,667 \mathrm{~cm}^{-1}$; Anal. Calcd for $\mathrm{C}_{35} \mathrm{H}_{28} \mathrm{~N}_{8} \mathrm{OS}_{2}$ : C, 65.60; $\mathrm{H}, 4.40 ; \mathrm{N}, 17.49$. Found: $\mathrm{C}, 65.62 ; \mathrm{H}, 4.39 ; \mathrm{N}, 17.51 ; \mathrm{HRMS}$ calcd for $\left(\mathrm{C}_{35} \mathrm{H}_{28} \mathrm{~N}_{8} \mathrm{OS}_{2}+\mathrm{H}^{+}\right)$: 641.1900; found: 641.1901.

2-(4-Methoxyphenyl)-5-(4-(6-(4-(5-(4-nitrophenyl)-1,3,4-thiadiazol-2-yl)phenyl)-1,2,4,5-tetrazin-3-yl)phenyl)1,3,4-thiadiazole (8i). Yellow solid (0.50 g, 44\% yield); mp 172-174 ${ }^{\circ} \mathrm{C} ;{ }^{1} \mathrm{H} \mathrm{NMR}\left(400 \mathrm{MHz}, \mathrm{DMSO}-d_{6}\right): \delta 3.87(\mathrm{~s}$, 3H), 7.13 (d, J $8.0 \mathrm{~Hz}, 2 \mathrm{H}), 7.17$ (d, J $8.0 \mathrm{~Hz}, 2 \mathrm{H}), 7.92$ (d, J $8.0 \mathrm{~Hz}, 2 \mathrm{H}), 8.04$ (d, J $8.0 \mathrm{~Hz}, 2 \mathrm{H}), 8.09(\mathrm{~d}, J 8.0 \mathrm{~Hz}$, $4 \mathrm{H}), 8.16-8.22(\mathrm{~m}, 2 \mathrm{H}), 8.28(\mathrm{~d}, J 8.0 \mathrm{~Hz}, 2 \mathrm{H}) ;{ }^{13} \mathrm{C}$ NMR $\left(100 \mathrm{MHz}, \mathrm{DMSO}-d_{6}\right): \delta 55.5,114.9,118.1,126.4,126.9$, $127.2,127.4,128.2,128.4,128.5,128.6,128.8,129.1,129.5,133.3,152.7,161.5,161.9,162.3,164.2,164.6$, 166.5, 166.9; UV-VIS: $\lambda_{\max }(\mathrm{MeOH}) 250 \mathrm{~nm}\left(\varepsilon \cdot 10^{-3}=26.54 \mathrm{~cm}^{-1} \mathrm{M}^{-1}\right), 312 \mathrm{~nm}\left(\varepsilon \cdot 10^{-3}=50.74 \mathrm{~cm}^{-1} \mathrm{M}^{-1}\right)$; fluorescence $\left(\mathrm{CH}_{3} \mathrm{OH}\right): \lambda_{\text {ex }}=318 \mathrm{~nm} ; \lambda_{\text {em }}=433 \mathrm{~nm}$; IR (ATR) v: 3363, 2229, 1675, 1608, 1551, 1519, 1493, 1442, 1413, 1308, 1253, 1174, 1100, 1070, 1026, 989, 962, 832, 773, 743, 703, $674 \mathrm{~cm}^{-1}$; Anal. Calcd for $\mathrm{C}_{31} \mathrm{H}_{19} \mathrm{~N}_{9} \mathrm{O}_{3} \mathrm{~S}_{2}: \mathrm{C}, 59.13 ; \mathrm{H}, 3.04 ; \mathrm{N}, 20.02$. Found: $\mathrm{C}, 59.15 ; \mathrm{H}, 3.03 ; \mathrm{N}, 20.04 ; \mathrm{HRMS}$ calcd for $\left(\mathrm{C}_{31} \mathrm{H}_{19} \mathrm{~N}_{9} \mathrm{O}_{3} \mathrm{~S}_{2}+\mathrm{H}^{+}\right)$: 630.1125; found: 630.1127.

\section{2-(4-(tert-Butyl)phenyl)-5-(4-(6-(4-(5-(4-nitrophenyl)-1,3,4-thiadiazol-2-yl)phenyl)-1,2,4,5-tetrazin-3-}

yl)phenyl)-1,3,4-thiadiazole (8j). Yellow solid (0.50 g, 42\% yield); mp 191-193 ${ }^{\circ} \mathrm{C} ;{ }^{1} \mathrm{H} \mathrm{NMR}(400 \mathrm{MHz}$, DMSO$\left.d_{6}\right): \delta 1.31(\mathrm{~s}, 9 \mathrm{H}), 7.53(\mathrm{~d}, J 8.0 \mathrm{~Hz}, 2 \mathrm{H}), 7.87(\mathrm{~d}, J 8.0 \mathrm{~Hz}, 2 \mathrm{H}), 8.00-8.03(\mathrm{~m}, 2 \mathrm{H}), 8.07(\mathrm{~d}, J 8.0 \mathrm{~Hz}, 2 \mathrm{H}), 8.11-$ $8.14(\mathrm{~m}, 2 \mathrm{H}), 8.31-8.34(\mathrm{~m}, 2 \mathrm{H}), 8.41(\mathrm{~d}, J 8.0 \mathrm{~Hz}, 2 \mathrm{H}), 8.46(\mathrm{~d}, J 12.0 \mathrm{~Hz}, 2 \mathrm{H}) ;{ }^{13} \mathrm{C} \mathrm{NMR}\left(100 \mathrm{MHz}, \mathrm{DMSO}-d_{6}\right): \delta$ 30.9, 34.7, 118.2, 124.6, 125.2, 126.1, 126.9, 127.3, 127.6, 127.9, 128.2, 128.5, 129.0, 129.3, 129.8, 133.3, 152.7, 154.6, 161.5, 163.3, 163.3, 163.7, 165.5, 166.7; UV-VIS: $\lambda_{\max }(\mathrm{MeOH}) 242 \mathrm{~nm}\left(\varepsilon \cdot 10^{-3}=34.62 \mathrm{~cm}^{-1} \mathrm{M}^{-1}\right)$, $337 \mathrm{~nm}\left(\varepsilon \cdot 10^{-3}=27.42 \mathrm{~cm}^{-1} \mathrm{M}^{-1}\right)$; fluorescence $\left(\mathrm{CH}_{3} \mathrm{OH}\right): \lambda_{\mathrm{ex}}=338 \mathrm{~nm} ; \lambda_{\mathrm{em}}=411 \mathrm{~nm}$; IR (ATR) v: 3343, 3234, 2947, 2356, 2229, 1671, 1635, 1608, 1582, 1548, 1492, 1444, 1414, 1364, 1341, 1305, 1279, 1179, 1122, 1027, 848, 769, 745, 704, $674 \mathrm{~cm}^{-1}$; Anal. Calcd for $\mathrm{C}_{34} \mathrm{H}_{25} \mathrm{~N}_{9} \mathrm{O}_{2} \mathrm{~S}_{2}$ : C, 62.28; $\mathrm{H}, 3.84 ; \mathrm{N}, 19.22$. Found: C, 62.29; $\mathrm{H}, 3.86$; $\mathrm{N}, 19.21$; HRMS calcd for $\left(\mathrm{C}_{34} \mathrm{H}_{25} \mathrm{~N}_{9} \mathrm{O}_{2} \mathrm{~S}_{2}+\mathrm{H}^{+}\right)$: 656.1645; found: 656.1644 .

\section{Acknowledgements}

The authors would like to thank the late Professor Rafał Kruszyński for his contribution to this project.

\section{Supplementary Material}

${ }^{1} \mathrm{H}$ and ${ }^{13} \mathrm{C}$ NMR, UV-Vis, and fluorescence spectra for compounds $\mathbf{6 a}-\mathbf{d}$ and $\mathbf{8 a}-\mathbf{j}$ are provided as supplementary material in the online version. 


\section{References}

1. Clavier, G.; Audebert, P. Chem Rev 2010, 110, 3299.

https://doi.org/10.1021/cr900357e

2. Saracoglu, N. Tetrahedron 2007, 63, 4199.

https://doi.org/10.1016/j.tet.2007.02.051

3. Gheno, A.; Trigaud, T.; Bouclé, J.; Audebert, P.; Ratier, B.; Vedraine S. Opt Mater (Amst) 2018, 75, 781. https://doi.org/10.1016/j.optmat.2017.11.027

4. Moral, M.; Garzón, A.; Olivier, Y.; Muccioli, L.; Sancho-García, J. C.; Granadino-Roldán, J. M.; FernándezGómez, M. J Phys Chem C 2015, 119, 18945.

https://doi.org/10.1021/acs.jpcc.5b05015

5. Miomandre, F.; Audebert, P. J Photochem Photobiol C 2020, 44, 100372.

https://doi.org/10.1016/j.jphotochemrev.2020.100372

6. Milad, R.; Essalah, K.; Abderrabba, M. J Phys Org Chem 2018, 31, 1.

https://doi.org/10.1002/poc.3750

7. Khudhair, A.M.; Ajeel, F.N.; Mohammed, M.H. Chem Phys Lett 2018, 713, 166.

https://doi.org/10.1016/i.cplett.2018.10.014

8. Xu, X.; Yu, T.; Bi, Z.; Ma, W.; Li, Y.; Peng, Q. Adv Mater 2018, 30, 1703973.

https://doi.org/10.1002/adma.201703973

9. Werbel, L.; McNamara, D.; Colbry, N.; Johnson, J.; Degan, M.; Whitney, B. J Heterocycl Chem 1979, 16, 881.

https://doi.org/10.1002/jhet.5570160511

10. Rusinov, G. L.; Latosh, N. I.; Ishmetova, R. I.; Kravchenko, M. A.; Ganebnykh, I. N.; Sokolov, V. A.; Chupakhin O. N Pharm Chem J 2005, 39, 8.

https://doi.org/10.1007/s11094-005-0068-1

11. Gadad, A.K.; Noolvi, M.N.; Karpoormath, R.V. Bioorg Med Chem 2004, 12, 5651.

https://doi.org/10.1016/i.bmc.2004.07.060

12. Mohan, J. Org Prep Proced Int 1992, 24, 523.

https://doi.org/10.1080/00304949209356719

13. Badr, S.M.I.; Barwa, R.M. Bioorg Med Chem 2011, 19, 4506.

https://doi.org/10.1016/j.bmc.2011.06.024

14. Hu, W. X.; Rao, G. W.; Sun, Y. Q. Bioorganic Med Chem Lett 2004, 14, 1177.

https://doi.org/10.1016/j.bmcl.2003.12.056

15. Cascioferro, S.; Li Petri, G.; Parrino, B.; El Hassouni, B.; Carbone, D.; Arizza, V.; Perricone, U.; Padova, A.;

Funel, N.; Peters, G.J.; Cirrincione, G.; Giovannetti, E.; Diana, P. Molecules 2020, 25, 329.

https://doi.org/10.3390/molecules25020329

16. Chen, Z.; Xu, W.; Liu, K.; Yang, S.; Fan, H.; Bhadury, P.S.; Hu, D.Y.; Zhang, Y. Molecules 2010, $15,9046$. https://doi.org/10.3390/molecules15129046

17. Khan, I.; Ali, S.; Hameed, S.; Rama, N.H.; Hussain, M.T.; Wadood, A.; Uddin, R.; Ul-Haq, Z.; Khan, A.; Ali, S.; Choudhary, M.I. Eur J Med Chem 2010, 45, 5200.

https://doi.org/10.1016/j.ejmech.2010.08.034

18. Rostom, S.A.F.; El-Ashmawy, I.M.; Abd El Razik, H.A.; Badr, M.H.; Ashour, H.M.A. Bioorg Med Chem 2009, $17,882$.

https://doi.org/10.1016/j.bmc.2008.11.035 
19. Serban, G. Acta Pharm 2020, 70, 259.

https://doi.org/10.2478/acph-2020-0031

20. Hua, X.; Liu, C.; Zhou, S.; Chen, M.; Xiong, L.; Li, Y.; Li, Z. Chem Res Chin Univ 2017, 33, 882. https://doi.org/10.1007/s40242-017-7147-1

21. Lv, M.; Liu, G.; Jia, M.; Xu, H. Bioorg Chem 2018, 81, 88.

https://doi.org/10.1016/i.bioorg.2018.07.034

22. Wu, H.; Alexander, S. C.; Jin, S.; Devaraj, N.K. J Am Chem Soc 2016, 138, 11429.

https://doi.org/10.1021/jacs.6b01625

23. Wang, M.; Svatunek, D.; Rohlfing, K.; Liu, Y.; Wang, H.; Giglio, B.; Yuan, H.; Wu, Z.; Li, Z. Fox, J. Theranostics 2016, 6, 887.

https://doi.org/10.7150\%2Fthno.14742

24. Qu, Y.; Pander, P.; Vybornyi, O.; Vasylieva, M.; Guillot, R.; Miomandre, F.; Dias, F.B.; Skabara, P.; Data, P.; Clavier, G.; Audebert, P. J Org Chem 2020, 85, 3407.

https://doi.org/10.1021/acs.joc.9b02817

25. Kędzia, A.; Kudelko, A.; Świątkowski, M.; Kruszyński, R. Dyes Pigm 2020, 172, 107865. https://doi.org/10.1016/i.dyepig.2019.107865

26. Kędzia, A.; Kudelko, A.; Świątkowski, M.; Kruszyński, R. Dyes Pigm 2020, 183, 108715.

https://doi.org/10.1016/j.dyepig.2020.108715

27. Brouwer, A. M. Pure. Appl Chem 2011, 83, 2213.

https://doi.org/10.1351/PAC-REP-10-09-31

28. Melhuish, W.H. J Phys Chem 1961, 65, 229.

https://doi.org/10.1021/j100820a009

29. Birks, J. B.; Dyson, D.J. Proc Roy Soc A 1963, 275, 135.

https://doi.org/10.1098/rspa.1963.0159

30. Shamsipur, M.; Chaichi, M.J. Spectrochim. Acta Part A Mol. Biomol. Spectrosc. 2005, 61, 1227. https://doi.org/10.1016/i.saa.2004.06.045 